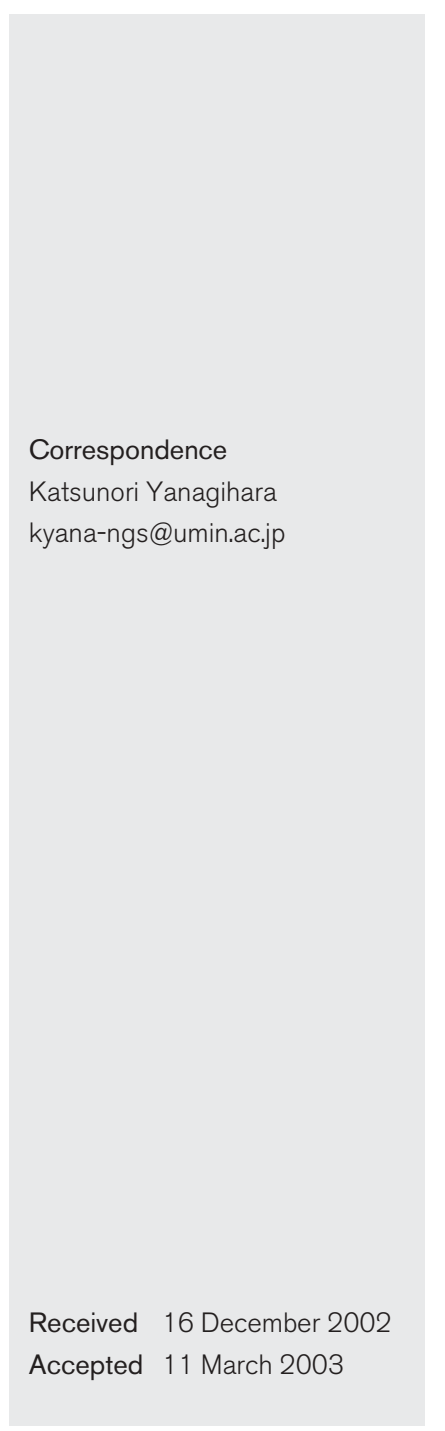

\title{
Role of elastase in a mouse model of chronic respiratory Pseudomonas aeruginosa infection that mimics diffuse panbronchiolitis
}

\author{
Katsunori Yanagihara, ${ }^{1,2}$ Kazunori Tomono, ${ }^{1}$ Yukihiro Kaneko, ${ }^{1}$ \\ Yoshitsugu Miyazaki, ${ }_{1}^{1}$ Kazuhiro Tsukamoto, ${ }^{1,2}$ Yoichi Hirakata, ${ }^{1}$ \\ Hiroshi Mukae, ${ }^{1}$ Jun-ichi Kadota, ${ }^{1}$ Ikuo Murata ${ }^{1,2}$ and Shigeru Kohno ${ }^{1,3}$ \\ ${ }^{1}$ Second Department of Internal Medicine, Nagasaki University School of Medicine, Nagasaki, \\ Japan \\ ${ }^{2}$ Department of Pharmacotherapeutics, Nagasaki University Graduate School of Phamaceutical \\ Sciences, Nagasaki, Japan \\ ${ }^{3}$ Division of Molecular and Clinical Microbiology, Department of Molecular Microbiology and \\ Immunology, Nagasaki University Graduate School of Medical Sciences, Nagasaki, Japan
}

\begin{abstract}
Pseudomonas aeruginosa frequently colonizes the respiratory tract of patients suffering from cystic fibrosis (CF) and diffuse panbronchiolitis (DPB). However, the relationship between lung inflammation and extracellular products of $P$. aeruginosa is not well-defined. To assess the role of elastase released by $P$. aeruginosa in DPB, a murine model of DPB was employed in this study. Mice were inoculated with either $P$. aeruginosa PAO1 or PAO-E64; the latter produces elastase with greatly reduced enzymic activity. Throughout the 90-day experiments, counts of viable bacteria from the PAO1- and PAO-E64-infected mice were found to be equivalent. However, the number of lymphocytes isolated from the lungs of PAO-E64-infected mice was significantly lower than the number isolated from the lungs of PAO1-infected animals. Histopathological examination of the lungs of mice infected by PAO1 on day 90 revealed an intense accumulation of chronic respiratory cells surrounding the bronchi, in sharp contrast to the more localized inflammatory response found in those mice infected by PAO-E64. These data suggest that $P$. aeruginosa elastase (PE) is a potent inflammatory factor in a mouse model of DPB and that the control of PE release by $P$. aeruginosa may be beneficial for patients with DPB.
\end{abstract}

\section{INTRODUCTION}

Pseudomonas aeruginosa is one of the most clinically relevant pathogens in patients with chronic respiratory conditions such as cystic fibrosis (CF) and diffuse panbronchiolitis (DPB). DPB is a clinicopathological entity characterized by chronic recurrent bronchiolitis and peribronchiolitis with infiltration of lymphocytes and plasma cells (Homma et al., 1983). P. aeruginosa is detected in $55 \%$ of patients with DPB on the first sputum culture, increasing to $82 \%$ during the late stage of DPB. The condition progresses insidiously and the prognosis, particularly after infection with $P$. aeruginosa, is considered to be poor.

$P$. aeruginosa virulence factors such as exotoxin A, exoenzyme S, elastase, alkaline protease, phospholipase C, LPS and phenazine pigments were found to be responsible for

Abbreviations: CF, cystic fibrosis; DPB, diffuse panbronchiolitis; PE, Pseudomonas aeruginosa elastase. damage to lung tissue by drawing parallels with acute $P$. aeruginosa infections. $P$. aeruginosa elastase (PE), a $39.5 \mathrm{kDa}$ metalloproteinase, is one of the strongest virulence factors among the toxins of this bacterium. It degrades the elastin of human lung and also other matrix proteins, including laminin and collagen types III and IV (Bejarano et al., 1989; Saulnier et al., 1989). It has tissue-damaging activity and destroys the structure of the lung. Experimental studies have also shown that PE is a potent inflammatory factor in the rat air-pouch inflammation model (Kon et al., 1999). Thus, we hypothesized that PE might play an important role in contributing to lung damage in DPB patients who become infected by $P$. aeruginosa.

In a series of recent studies, we have established a murine model of chronic $P$. aeruginosa respiratory tract infection that mimics DPB, and have investigated both the mechanisms of chronic infection and the effects of macrolide treatment using this model system (Yanagihara et al., 1997, 2000a, b, 2002). To test the hypothesis that elastase has a 
significant impact on DPB patients infected by $P$. aeruginosa, we challenged our murine model with low- or high-PEproducing strains of $P$. aeruginosa to assess the role of this enzyme in chronic inflammation.

\section{METHODS}

Laboratory animals. Male, 7-week-old, 30-35 g body weight, specific pathogen-free ddY mice were purchased from the Shizuoka Agricultural Cooperative Association for Laboratory Animals (Shizuoka, Japan). All animals were housed in a pathogen-free environment and received sterile food and water in the Laboratory Animal Center for Biomedical Science at Nagasaki University. The experimental protocol was approved by the Ethics Review Committee for Animal Experimentation at our institution.

Bacterial strains. $P$. aeruginosa strains $\mathrm{PAO} 1$ and $\mathrm{PAO}-\mathrm{E} 64$ were kindly provided by Professor B. H. Iglewski, University of Rochester School of Medicine and Dentistry, Rochester, NY, USA. Strain PAO1 has been well-characterized; it produces most of the recognized virulence factors (Holloway et al., 1979). Its elastase-mutant strain, PAO-E64 (obtained by nitrosoguanidine treatment), produces an elastase that is antigenically indistinguishable from that of the parent strain but has greatly reduced enzymic activity. The bacteria were stored at $-70{ }^{\circ} \mathrm{C}$ in brain-heart infusion broth (BBL) supplemented with $10 \%$ (v/v) glycerol and $5 \%(\mathrm{w} / \mathrm{v})$ skimmed milk until use.

Experimental model of chronic respiratory infection. Disposable sterile plastic cut-down intravenous catheters with $3 \mathrm{Fr}(1 \mathrm{~mm})$ outer diameter (Atom Medical Corporation, Tokyo) were used for intubation. The tube was $3.0 \mathrm{~mm}$ long; a few slits were made at the proximal end to prevent blockage by oral secretions. To prepare the inoculum, $P$. aeruginosa was cultured on trypticase soy agar plates for $24 \mathrm{~h}$. The bacteria were suspended in saline, harvested by centrifugation $(3000 \mathrm{~g}$ $4{ }^{\circ} \mathrm{C}, 10 \mathrm{~min}$ ), resuspended in sterile saline and adjusted to $1 \sim 2 \times 10^{8}$ c.f.u. $\mathrm{ml}^{-1}$, as estimated by turbidimetry. The intubation tube was then immersed in the bacterial saline suspension for 3 days at $37^{\circ} \mathrm{C}$. On day 3 post-inoculation, just prior to intubation, bacterial counts from these tubes were found to be $6 \cdot 0 \pm 0 \cdot 6\left(\log _{10}\right.$ c.f.u. $\mathrm{ml}^{-1}$, mean $\left.\pm \mathrm{SD}, n=10\right)$. The method used for inducing infection has been previously described in detail (Yanagihara et al., 1997).

Bacteriological and histopathological analysis. Both lungs were homogenized and cultured separately. Bacterial counts were performed by serial dilution on trypticase soy agar before being poured onto $\mathrm{N}$ acetyl-L-cysteine (NAC) agar plates (BBL). For histological examination, lung specimens were fixed in buffered formalin solution (10\%).

Preparation of pulmonary intraparenchymal lymphocytes. Pulmonary intraparenchymal lymphocytes were prepared as described previously (Abraham et al., 1990; Yanagihara et al., 1997). Briefly, mice were killed by cervical dislocation. After thoracotomy, the lung vascular bed was flushed by injecting $2-3 \mathrm{ml}$ chilled physiological saline into the right ventricle. The lungs were then excised, washed in physiological saline, teased with a stainless steel mesh and incubated in RPMI 1640 medium (Gibco-BRL) with $5 \%$ fetal calf serum, $100 \mathrm{U}$ penicillin $\mathrm{G} \mathrm{ml}^{-1}, 100 \mu \mathrm{g}$ streptomycin $\mathrm{ml}^{-1}, 10 \mathrm{mM}$ HEPES, $50 \mu \mathrm{M}$ 2-mercaptoethanol and $2 \mathrm{mM}$ L-glutamine, containing $20 \mathrm{U}$ collagenase $\mathrm{ml}^{-1}$ and $1.0 \mu \mathrm{g}$ DNase I ml ${ }^{-1}$ (both from Sigma). A volume of $10 \mathrm{ml}$ was used for each set of lungs. After incubation for $60 \mathrm{~min}$ at $37^{\circ} \mathrm{C}$ with vigorous shaking, the tissue fragments and most dead cells were removed by passage through $100 \mu \mathrm{m}$ nylon mesh. After centrifugation at $600 \mathrm{~g}$ for $5 \mathrm{~min}$ at $15^{\circ} \mathrm{C}$, the cell pellet was resuspended in $4 \mathrm{ml} 40 \%$ (v/v) Percoll (Pharmacia) and layered onto $4 \mathrm{ml} 80 \%$ (v/v) Percoll. After centrifugation at $600 \mathrm{~g}$ for $20 \mathrm{~min}$ at $15^{\circ} \mathrm{C}$, the cells at the interface were collected, washed twice with physiological saline and the number of monocytes was counted using a haemocytometer. Approximately $4 \times 10^{4}$ cells were centrifuged onto a glass slide at $160 \mathrm{~g}$ for $2 \mathrm{~min}$ using a Cytospin 2 centrifuge (Shandon Instruments) and stained by MayGiemsa staining. At least 300 cells were examined by photomicroscopy for differentiation of cellular fractions.

Statistical analysis. Data are expressed as mean \pm SD. Differences between groups were examined for statistical significance using Student's unpaired $t$-test. $P<0.05$ denoted the presence of a statistically significant difference.

\section{RESULTS}

\section{Bacteriological viable counts}

The mean c.f.u. $\pm \mathrm{SD}$ of $P$. aeruginosa recovered from homogenized lung tissue at various time intervals after inoculation are shown in Table 1. Six animals from each group were killed at 7, 30, 60 and 90 days after challenge. The mean numbers of viable bacteria from the lungs of PAO1and PAO-E64-infected mice were similar, and were stable at $10^{5}-10^{6}$ c.f.u. per lung up to 90 days after inoculation. The experiments were reproducible $(n=3)$ and representative data are shown.

\section{Histopathological examination}

Histopathological examination of the lungs of mice infected by strain PAO1 on day 90 post-intubation revealed an intense accumulation of chronic respiratory cells surrounding the bronchi (Fig. 1a, b). However, the lungs of mice that had been infected by strain PAO-E64 showed only a localized inflammatory process (Fig. 1c, d).

\section{Accumulation of lymphocytes in the lungs}

In mice infected by strain PAO1, the total number of lymphocytes in the lung had increased significantly by day 7 post-intubation, compared with lymphocyte numbers prior to intubation. Furthermore, this level was sustained throughout the 90 days of the experiment (Table 2). Lymphocyte numbers in PAO1-infected animals on day 90 were approximately 3-4-fold greater than had been found prior to intubation. In contrast, the total number of lymphocytes in the lungs of mice infected by strain PAO-

Table 1. Viable counts of $P$. aeruginosa from the lungs of mice infected by strain PAO1 or PAO-E64

Values are $\log _{10}$ c.f.u. P. aeruginosa $\mathrm{ml}^{-1}$, given as means \pm SD.

\begin{tabular}{|lcc|}
\hline $\begin{array}{l}\text { Time post-inoculation } \\
\text { (days) }\end{array}$ & PAO1 $(\boldsymbol{n}=\mathbf{6})$ & PAO-E64 $(\boldsymbol{n}=\mathbf{6})$ \\
\hline 0 & $6 \cdot 1 \pm 0 \cdot 8$ & $6 \cdot 3 \pm 1 \cdot 0$ \\
7 & $5 \cdot 4 \pm 0 \cdot 5$ & $5 \cdot 5 \pm 0 \cdot 4$ \\
30 & $5 \cdot 0 \pm 0 \cdot 6$ & $5 \cdot 8 \pm 0 \cdot 5$ \\
60 & $6 \cdot 1 \pm 0 \cdot 4$ & $5 \cdot 2 \pm 0 \cdot 4$ \\
90 & $5 \cdot 7 \pm 0 \cdot 5$ & $5 \cdot 3 \pm 0 \cdot 7$ \\
\hline
\end{tabular}



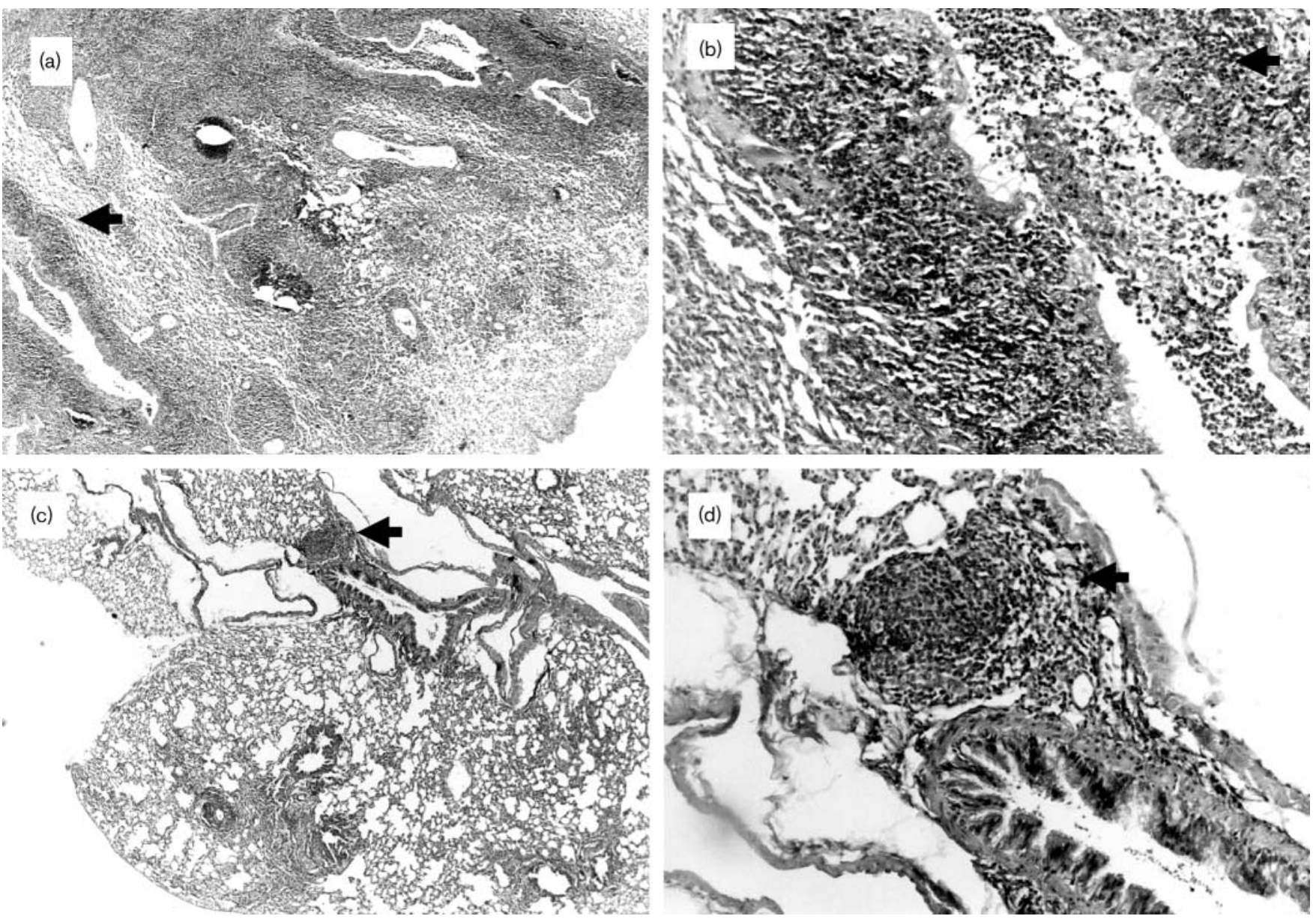

Fig. 1. Histopathological examination (haematoxylin-eosin stain) of lungs infected by PAO1 ( $a$, b; original magnification $\times 12$, $\times 100)$ and PAO-E64 (c, d; original magnification $\times 12, \times 100)$ on day $90 .(a, b)$ Lungs from mice infected by $P$. aeruginosa strain PAO1; intense accumulation of mononuclear inflammatory cells surrounding the bronchi was observed. (c, d) Lung specimens infected by PAO-E64, showing localized mononuclear inflammatory cells (arrows).

E64 did not increase significantly after inoculation and was considerably lower than in the lungs of animals infected by strain PAO1 throughout the study period (Table 2). The experiments were reproducible $(n=3)$ and representative data are shown.

\section{DISCUSSION}

Although $P$. aeruginosa frequently colonizes the respiratory tract of patients suffering from DPB and CF, the relationship between lung inflammation and the production of extracellular factors by this organism is not well-defined. Amitani et al. (1991) showed that PE contributed to epithelial damage in vitro. However, the epithelial cell damage was induced by neutrophil-derived, rather than Pseudomonas-derived, proteases found in the sputum of CF patients (Doring, 1994; Venaille et al., 1998). Resistance of human tracheal epithelial cells to killing by PE has also been reported (Kercsmar \&
Davis, 1993). These in vitro studies suggested that the effect of $\mathrm{PE}$ on respiratory infection was still controversial and that an in vivo study was needed to clarify this issue.

Thus, a murine model that mimics DPB was employed to reveal the relationship between $\mathrm{PE}$ and lung inflammation in patients with DPB. Viable bacteria were regularly isolated from the lungs for more than 1 year in this model. The histopathological features, which consisted of massive accumulation of lymphocytes in the lung, also resembled those of DPB (Yanagihara et al., 1997). The present study demonstrates that elastase released by $P$. aeruginosa plays an important role in DPB and causes marked inflammation. While counts of viable bacteria from the lungs of PAO1- and PAO-E64-infected mice were similar, the numbers of lymphocytes in the lungs of the PAO-E64-infected animals were significantly lower than those in mice infected by PAO1. We quantified the number of lymphocytes in the whole lung, as this parameter is a good marker of the degree of chronic 
Table 2. Numbers of lymphocytes isolated from the lungs of mice infected by $P$. aeruginosa strain PAO1 or PAO-E64

Values are number of lymphocytes in the lung $\left(\times 10^{5}\right)$, given as means \pm SD.

\begin{tabular}{|lrc|}
\hline $\begin{array}{l}\text { Time post-inoculation } \\
\text { (days) }\end{array}$ & PAO1 $(\boldsymbol{n}=\mathbf{5})$ & PAO-E64 $(\boldsymbol{n}=\mathbf{5})$ \\
\hline 0 & $5 \cdot 1 \pm 0 \cdot 9$ & $4 \cdot 6 \pm 1 \cdot 0$ \\
7 & $13 \cdot 4 \pm 2 \cdot 5$ & $6 \cdot 5 \pm 3 \cdot 4^{*}$ \\
30 & $21 \cdot 3 \pm 2 \cdot 6$ & $6 \cdot 8 \pm 2 \cdot 5^{*}$ \\
60 & $16 \cdot 9 \pm 3 \cdot 4$ & $7 \cdot 2 \pm 1 \cdot 4^{*}$ \\
90 & $19 \cdot 7 \pm 4 \cdot 5$ & $6 \cdot 3 \pm 2 \cdot 7^{*}$ \\
\hline
\end{tabular}

${ }^{\star} P<0 \cdot 01$ versus mice infected by $\mathrm{PAO} 1$.

inflammation. Lymphocyte numbers in PAO1-infected mice on day 90 were approximately 3-4-fold greater than those prior to intubation. These results indicate that PE contributed to lymphocyte accumulation in the lungs of mice with chronic Pseudomonas infection. The data from our study are supported by those of Woods et al. (1982), who suggested that PE contributed to lung inflammation in rats. Azghani et al. (2002) reported that PE induces phosphorylation of the extracellular signal-regulated (ERK1/2) proteins of the MAPK pathway in A549 epithelial cells. This report suggested that the PE may augment pulmonary inflammation via cellular signalling.

A similar process may operate in patients with DPB. A number of studies in vitro, including those from our laboratories, have suggested that macrolides can inhibit extracellular protease enzymes produced by $P$. aeruginosa without affecting bacterial proliferation (Hirakata et al., 1992; Sakata et al., 1993; Mizukane et al., 1994). These results suggest a relationship between clinical improvement by macrolide treatment (Kudoh et al., 1987, 1998; Yanagihara et al., 2001) and reduction of elastase production by $P$. aeruginosa, in patients with DPB. Similarly, we reported previously that treatment with erythromycin resulted in clinical improvement in patients with DPB, independently of $P$. aeruginosa infection (Fujii et al., 1995). These results correlate with studies in vitro, which show macrolideinduced inhibition of elastase production by $P$. aeruginosa without any effect on bacterial proliferation (Sakata et al., 1993). Our data also support a report that immunization with a PE peptide can reduce the severity of lung infections due to $P$. aeruginosa (Sokol et al., 2000).

In conclusion, our results suggest that $\mathrm{PE}$ is a potent inflammatory mediator in a mouse model of DPB, and that $\mathrm{PE}$ inhibition via macrolide treatment may result in the clinical improvement of patients affected by this condition.

\section{ACKNOWLEDGEMENTS}

The authors thank Professor B. H. Iglewski (University of Rochester
School of Medicine and Dentistry, Rochester, NY, USA) for providing $P$. aeruginosa strains PAO1 and PAO-E64, and Dr F. G. Issa (Word-Medex, Sydney, Australia) for his assistance with editing the manuscript.

\section{REFERENCES}

Abraham, E., Freitas, A. A. \& Coutinho, A. A. (1990). Purification and characterization of intraparenchymal lung lymphocytes. J Immunol 144, 2117-2122.

Amitani, R., Wilson, R., Rutman, A., Read, R., Ward, C., Burnett, D., Stockley, R. A. \& Cole, P. J. (1991). Effects of human neutrophil elastase and Pseudomonas aeruginosa proteinases on human respiratory epithelium. Am J Respir Cell Mol Biol 4, 26-32.

Azghani, A. O., Baker, J. W., Shetty, S., Miller, E. J. \& Bhat, G. J. (2002). Pseudomonas aeruginosa elastase stimulates ERK signaling pathway and enhances IL-8 production by alveolar epithelial cells in culture. Inflamm Res 51, 506-510.

Bejarano, P. A., Langeveld, J. P., Hudson, B. G. \& Noelken, M. E. (1989). Degradation of basement membranes by Pseudomonas aeruginosa elastase. Infect Immun 57, 3783-3787.

Doring, G. (1994). The role of neutrophil elastase in chronic inflammation. Am J Respir Crit Care Med 150, S114-S117.

Fujii, T., Kadota, J., Kawakami, K., lida, K., Shirai, R., Kaseda, M., Kawamoto, S. \& Kohno, S. (1995). Long term effect of erythromycin therapy in patients with chronic Pseudomonas aeruginosa infection. Thorax 50, 1246-1252.

Hirakata, Y., Kaku, M., Mizukane, R., Ishida, K., Furuya, N., Matsumoto, T., Tateda, K. \& Yamaguchi, K. (1992). Potential effects of erythromycin on host defense systems and virulence of Pseudomonas aeruginosa. Antimicrob Agents Chemother 36, 1922-1927.

Holloway, B. W., Krishnapillai, V. \& Morgan, A. F. (1979). Chromosomal genetics of Pseudomonas. Microbiol Rev 43, 73-102.

Homma, H., Yamanaka, A., Tanimoto, S., Tamura, M., Chijimatsu, Y., Kira, S. \& Izumi, T. (1983). Diffuse panbronchiolitis. A disease of the transitional zone of the lung. Chest 83, 63-69.

Kercsmar, C. M. \& Davis, P. B. (1993). Resistance of human tracheal epithelial cells to killing by neutrophils, neutrophil elastase, and Pseudomonas elastase. Am J Respir Cell Mol Biol 8, 56-62.

Kon, Y., Tsukada, H., Hasegawa, T., Igarashi, K., Wada, K., Suzuki, E., Arakawa, M. \& Gejyo, F. (1999). The role of Pseudomonas aeruginosa elastase as a potent inflammatory factor in a rat air pouch inflammation model. FEMS Immunol Med Microbiol 25, 313-321.

Kudoh, S., Uetake, T., Hagiwara, K., Hirayama, M., Hus, L. H., Kimura, H. \& Sugiyama, Y. (1987). Clinical effects of low-dose long-term erythromycin chemotherapy on diffuse panbronchiolitis. Nihon Kyobu Shikkan Gakkai Zasshi (Jpn J Thorac Dis) 25, 632-642 (in Japanese).

Kudoh, S., Azuma, A., Yamamoto, M., Izumi, T. \& Ando, M. (1998). Improvement of survival in patients with diffuse panbronchiolitis treated with low-dose erythromycin. Am J Respir Crit Care Med 157, 1829-1832.

Mizukane, R., Hirakata, Y., Kaku, M., Ishii, Y., Furuya, N., Ishida, K., Koga, H., Kohno, S. \& Yamaguchi, K. (1994). Comparative in vitro exoenzyme-suppressing activities of azithromycin and other macrolide antibiotics against Pseudomonas aeruginosa. Antimicrob Agents Chemother 38, 528-533.

Sakata, K., Yajima, H., Tanaka, K., Sakamoto, Y., Yamamoto, K., Yoshida, A. \& Dohi, Y. (1993). Erythromycin inhibits the production of elastase by Pseudomonas aeruginosa without affecting its proliferation in vitro. Am Rev Respir Dis 148, 1061-1065.

Saulnier, J. M., Curtil, F. M., Duclos, M. C. \& Wallach, J. M. (1989). Elastolytic activity of Pseudomonas aeruginosa elastase. Biochim Biophys Acta 995, 285-290. 
Sokol, P. A., Kooi, C., Hodges, R. S., Cachia, P. \& Woods, D. E. (2000). Immunization with a Pseudomonas aeruginosa elastase peptide reduces severity of experimental lung infections due to $P$. aeruginosa or Burkholderia cepacia. J Infect Dis 181, 1682-1692.

Venaille, T. J., Ryan, G. \& Robinson, B. W. (1998). Epithelial cell damage is induced by neutrophil-derived, not pseudomonas-derived, proteases in cystic fibrosis sputum. Respir Med 92, 233-240.

Woods, D. E., Cryz, S. J., Friedman, R. L. \& Iglewski, B. H. (1982). Contribution of toxin A and elastase to virulence of Pseudomonas aeruginosa in chronic lung infections of rats. Infect Immun 36, $1223-1228$.

Yanagihara, K., Tomono, K., Sawai, T., Hirakata, Y., Kadota, J., Koga, H., Tashiro, T. \& Kohno, S. (1997). Effect of clarithromycin on lymphocytes in chronic respiratory Pseudomonas aeruginosa infection. Am J Respir Crit Care Med 155, 337-342.
Yanagihara, K., Tomono, K., Sawai, T. \& 10 other authors (2000a). Combination therapy for chronic Pseudomonas aeruginosa respiratory infection associated with biofilm formation. J Antimicrob Chemother 46, 69-72.

Yanagihara, K., Tomono, K., Kuroki, M. \& 8 other authors (2000b). Intrapulmonary concentrations of inflammatory cytokines in a mouse model of chronic respiratory infection caused by Pseudomonas aeruginosa. Clin Exp Immunol 122, 67-71.

Yanagihara, K., Kadoto, J. \& Kohno, S. (2001). Diffuse panbronchiolitis - pathophysiology and treatment mechanisms. Int J Antimicrob Agents 18, S83-S87.

Yanagihara, K., Tomono, K., Imamura, Y. \& 8 other authors (2002). Effect of clarithromycin on chronic respiratory infection caused by Pseudomonas aeruginosa with biofilm formation in an experimental murine model. J Antimicrob Chemother 49, 867-870. 